\title{
Docosahexaenoic Acid Induces Adipose Differentiation-Related Protein through Activation of Retinoid X Receptor in Human Choriocarcinoma BeWo Cells
}

\author{
Kazushige Suzuki,${ }^{a, b}$ Katsuhiko Takahashi,${ }^{c}$ Tomoko Nishimaki-Mogami ${ }^{d}$ Hiroyuki Kagechika, ${ }^{e}$ \\ Matsuo Yамамото, ${ }^{b}$ and Hiroyuki $\operatorname{ITABE}^{*, a}$ \\ ${ }^{a}$ Department of Biological Chemistry, Showa University School of Pharmacy; 1-5-8 Hatanodai, Shinagawa-ku, Tokyo \\ 142-8555, Japan: ${ }^{b}$ Department of Periodontology, Showa University School of Dentistry; 2-1-2 Kitasenzoku, Ota-ku, \\ Tokyo 145-8515, Japan: ' Laboratory of Physiological Chemistry, Institute of Medicinal Chemistry, Hoshi University; \\ 2-4-41 Ebara, Shinagawa-ku, Tokyo 142-8501, Japan: ' National Institute of Health Sciences; 1-18-1 Kamiyoga, \\ Setagaya-ku, Tokyo 158-8501, Japan: and ${ }^{e}$ School of Biomedical Science, Institute of Biomaterials and Bioengineering, \\ Tokyo Medical and Dental University; 2-3-10 Kanda-Surugadai, Chiyoda-ku, Tokyo 101-0062, Japan.
}

Received February 5, 2009; accepted April 2, 2009; published online April 9, 2009

\begin{abstract}
Adipose differentiation-related protein (ADRP) is associated with intracellular lipid droplets that accumulate neutral lipids. Here we report that ADRP expression in a human choriocarcinoma cell line, BeWo, is regulated through activation of retinoid $X$ receptor $(\mathrm{RXR})$ and peroxisome proliferator-activated receptor- $\gamma(\operatorname{PPAR} \gamma)$. Incubation with docosahexaenoic acid (DHA) or oleic acid (OA) induced accumulation of triacylglycerol (TG) and ADRP in BeWo cells. DHA-induced ADRP expression was suppressed by RXR-antagonists, PA452 and HX531. However, oleic acid-induced ADRP expression was not blocked by the RXR-antagonists but by a PPAR $\gamma$ antagonist. Treatment of the cells with RXR-agonists, HX630 and PA024, increased Adrp transcripts, however, they alone did not change the levels of ADRP protein and TG in BeWo cells. Induction of ADRP protein was observed in the presence of a proteasome inhibitor, suggesting that ADRP is degraded under lipid-poor conditions. These results suggest that expression of ADRP is in part regulated by RXR and PPAR $\gamma$ transcription factors, and DHA induces ADRP by acting as an endogenous agonist of RXR.
\end{abstract}

Key words docosahexaenoic acid; retinoid X receptor; lipid droplet; BeWo cell; adipose differentiation-related protein; peroxisome proliferator-activated receptor- $\gamma$

Intracellular lipid droplet (LD) is a cellular organelle observed ubiquitously in many cell types including chorionic cells. LDs are storage sites for neutral lipids and may play a central role in whole body energy homeostasis and distribution of lipids. ${ }^{1,2)}$ Adipose differentiation-related protein (ADRP; also called as ADFP or adipophilin) is a major LDforming protein expressed in a wide variety of cells and tissues including placenta. ${ }^{3-7)}$ Addition of high concentrations of fatty acids to various cells in culture up-regulates both Adrp mRNA and ADRP protein, ${ }^{8)}$ while ADRP is degraded via the ubiquitin-proteasome mechanism when cellular triacylglycerol (TG) is reduced. $\left.{ }^{9}, 10\right)$ These observations suggest that ADRP has a role in cellular fatty acid uptake and storage.

Placental transport of fatty acids to the fetus is essential for proper fetal growth and development. Fetal demand for fatty acids increases during the latter stages of pregnancy, and long chain $n-3$ polyunsaturated fatty acids (LCPUFA) are supplied from the maternal circulation. ${ }^{11)}$ Docosahexaenoic acid (DHA: $22: 6, n-3$ ) plays a particularly important role in fetal development because it is a major lipid constituent in the brain and retina. ${ }^{12,13)}$ The blood DHA concentration is higher in the fetus than in maternal circulation, suggesting an active placental transfer. ${ }^{12)}$ In BeWo cells, a human placental choriocarcinoma cell line, DHA was preferentially incorporated into TG. ${ }^{14)}$ From postmortem studies of fetuses, stillbirths, and preterm infants, it was estimated that the fetus consumes $<50-60 \mathrm{mg} / \mathrm{d}$ of $\mathrm{n}-3$ LCPUFA during the last trimester of pregnancy, which are probably transferred from the placenta. ${ }^{15)}$

Peroxisome proliferator-activated receptor- $\gamma(\operatorname{PPAR} \gamma)$ is a nuclear receptor that acts as a transcription factor by forming a heterodimer with its partner, retinoid $\mathrm{X}$ receptor (RXR). Both PPAR- $\gamma$ and RXR are essential for proper placental development, since disruption of these genes in mice resulted in embryonic death, with embryos exhibiting placental defects, including improper labyrinth development and reduced LD in trophoblasts. ${ }^{16,17)}$ Sadovsky and colleagues demonstrated that PPAR $\gamma$ enhances the differentiation of cultured primary term human trophoblasts, and that PPAR $\gamma$ increases LDs in syncytiotrophoblasts. ${ }^{18)}$ Since it has been suggested that DHA is a natural ligand of RXR, ${ }^{19,20)}$ we assumed that DHA plays a role in placental development through ADRP expression and $\mathrm{LD}$ formation.

In this study, we examined DHA-dependent ADRP expression in BeWo cells. BeWo cells, a human placental choriocarcinoma cell line, maintain several characteristics of natural trophoblasts. We showed that DHA induces Adrp mRNA expression and ADRP protein accumulation in BeWo cells. The DHA-dependent ADRP accumulation was inhibited by RXR-antagonists, indicating that DHA acts as a natural RXR-agonist to induce ADRP.

\section{MATERIALS AND METHODS}

Cell Culture BeWo cells were cultured in Ham's F12 medium with $15 \%$ fetal bovine serum (FBS) supplemented with $2 \mathrm{mM} \mathrm{L}$-glutamine and $1 \%$ antibiotics $(50 \mathrm{U} / \mathrm{ml}$ penicillin and $50 \mu \mathrm{g} / \mathrm{ml}$ streptomycin). The cells were maintained at $37^{\circ} \mathrm{C}$ in a $5 \% \mathrm{CO}_{2}$ atmosphere. Cells were supplemented with $100 \mu \mathrm{M}$ DHA or oleic acid (OA) to induce LD formation. Stock solutions of $4 \mathrm{~mm}$ DHA and OA were prepared as 
complexes with BSA as previously described. ${ }^{9)}$ To examine the effect of nuclear receptors, PA024 and HX630 (RXR agonist), ${ }^{21,22)}$ PA452 and HX531 (RXR antagonist), ${ }^{23,24)}$ GW1929 (PPAR $\gamma$ agonist; Sigma), and GW9662 (PPAR $\gamma$ antagonist; Alexis) were used. These reagents dissolved in dimethylsufoxide were added to the cells $(10 \mu \mathrm{l} \mathrm{DMSO} / 5 \mathrm{ml}$ cell culture dish). As a control experiment, cells were treated with DMSO without the reagents under the same conditions.

Real-Time Polymerase Chain Reaction (PCR) Total RNA from BeWo cells $\left(1 \times 10^{6} /\right.$ dish $)$ was extracted with ISOGEN (Nippongene; Toyama, Japan) and reverse-transcribed with a Reverse Transcription Kit (Qiagen). Real time-PCR was performed using a SYBR Green I Master RT-PCR kit equipped with a LightCycler (Roche). The following primers were used: ADRP, 5'-AGTGGAAAAGGAGCATTGGA-3' and 5'-AGTTGAGGGGCGTGTTGTTA-3'; 18S, 5'-CGCCGCTAGAGGTGAAATTCT-3' and 5'-CGAACCTCCGACTTTCGTTCT-3'. The PCR conditions were $95^{\circ} \mathrm{C}$ for $10 \mathrm{~min}$ followed by 40 cycles of $10 \mathrm{~s}$ at $95^{\circ} \mathrm{C}, 10 \mathrm{~s}$ at $60^{\circ} \mathrm{C}$, and $8 \mathrm{~s}$ at $72{ }^{\circ} \mathrm{C}$. The number of transcripts was quantified, and each sample was normalized on the basis of $18 \mathrm{~S}$ content.

Immunoblot BeWo cells were homogenized in a buffer containing $1 \%$ Triton $\mathrm{X}-100$, and the homogenate was centrifuged at $14000 \mathrm{rpm}$ for $10 \mathrm{~min}$ at $4{ }^{\circ} \mathrm{C}$. Protein concentration was determined using the $\mathrm{BCA}$ protein assay reagent (Pierce; Rockford, IL, U.S.A.) with BSA as the standard. The cell lysates $(20 \mu \mathrm{g}$ protein) were separated by $10 \%$ sodium dodecyl sulfate-polyacrylamide gel electrophoresis (SDSPAGE) and then electrotransferred onto a nitrocellulose membrane (Protran BA-83; Schleicher and Schuell). The membrane was blocked with TBS containing 3\% BSA and then incubated for $1 \mathrm{~h}$ with antibody against ADRP (American Research Products 03-610102; Belmont, MA, U.S.A.), after which the positive bands were visualized with horseradish peroxidase (HRP)-conjugated anti-mouse immunoglobulin $\mathrm{G}(\mathrm{IgG})$ and an ECL Western blot detection kit (Perkin
Elmer) and detected using X-ray film (RX-U; Fuji Film). The same membrane was reprobed with antibody against GAPDH (MAB374; Chemicon Inc.) or antibody against TIP47 (Santa Cruz Biotechnology). The intensities of bands were analysed using Scion Image analysis software.

Fluorescence Microscopy BeWo cells were cultured on chamber slides and were fixed with $2 \%$ formaldehyde in PBS for $20 \mathrm{~min}$ at room temperature and stained with Oil Red O to visualize neutral LD. After washing with PBS, the cells were incubated with anti-ADRP monoclonal antibody (dilution of $1: 100$ in PBS containing $0.1 \%$ BSA, and $0.1 \%$ saponin) followed by Alexa488-labeled goat anti-(mouse IgG) antibody (dilution $1: 100)$, and nuclei were stained with DAPI.

Other Methods The amounts of TG were determined enzymatically using TG-test Wako (Wako Chemical Co., Osaka, Japan). Data are expressed as means \pm standard deviation. Results were analyzed using Student's $t$-test, and statistical significance was assigned at $p<0.05$.

\section{RESULTS}

Effects of DHA on ADRP mRNA and Protein Expression Since DHA is a crucial nutrient for fetal development, we questioned whether DHA has an effect on lipid mobilization in placental cells. Incubation of BeWo cells with $100 \mu \mathrm{M}$ DHA for up to $48 \mathrm{~h}$ increased the cellular amounts of ADRP protein and TG in a time-dependent manner (Figs. 1A, B). Under the same conditions, Adrp mRNA expression was upregulated for about 5-fold by treatment with DHA for $24 \mathrm{~h}$. The effect of DHA on induction of Adrp mRNA was more efficient than OA treatment (Fig. 1C). DHA and OA increased intracellular ADRP protein and TG about 30- and 2fold, respectively (Figs. 1D, E). The difference of mRNA level induced by these fatty acids was not directly correlated with the amount of ADRP protein, presumably because the
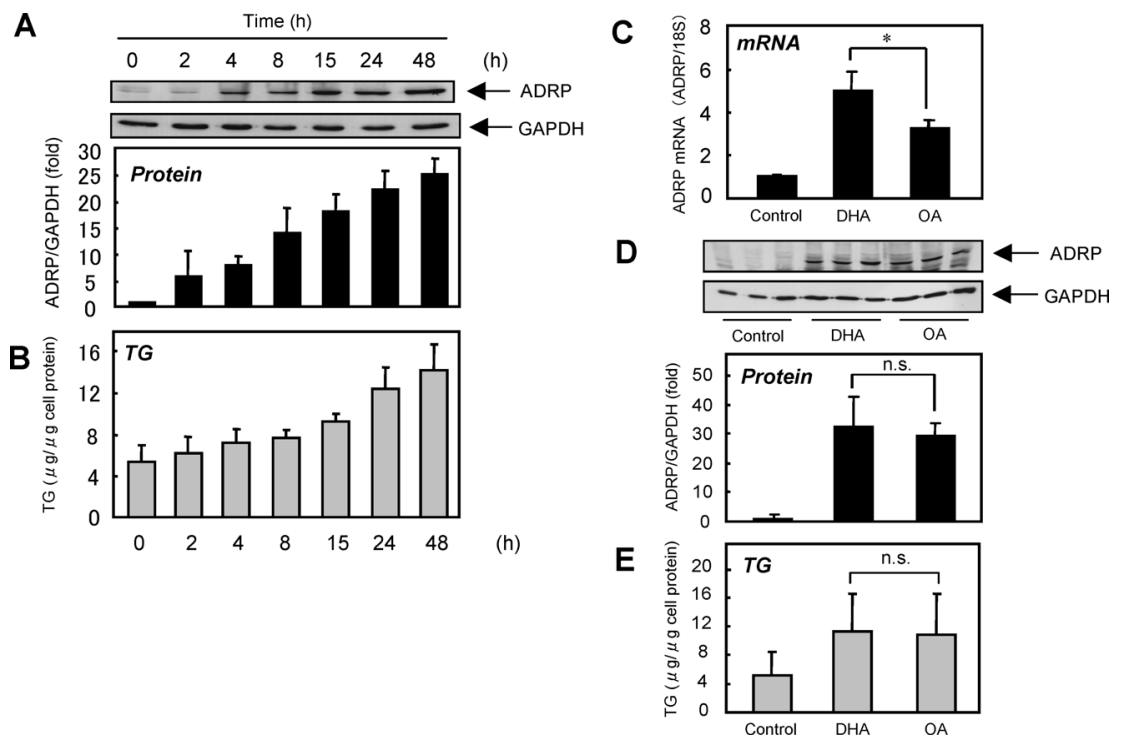

Fig. 1. DHA and OA Increased ADRP Expression in BeWo Cells

(A) BeWo cells were incubated with $100 \mu \mathrm{M}$ DHA for up to $48 \mathrm{~h}$ in serum-containing medium. The cell lysates were subjected to immunoblotting to detect ADRP and GAPDH, and the amounts of TG (B) in the cell lysates were measured using the enzymatic assay. (C) BeWo cells were incubated with $100 \mu \mathrm{M}$ DHA or OA for $24 \mathrm{~h}$ in serum-containing medium. Total RNA was extracted from the cells and real-time PCR experiments were carried out to evaluate the amount of mRNAs for ADRP and 18S. Values are reported as the amount of Adrp mRNA relative to 18S mRNA. (D) ADRP and GAPDH in the cell lysates were detected by immunoblotting. (E) The amounts of TG in the cell lysates were measured using the enzymatic assay. * Statistically significant $(p<0.05)$ between DHA and OA. n.s.: not significant. 


\section{A}

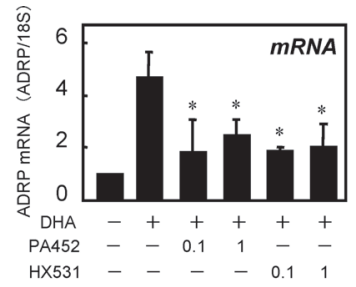

B

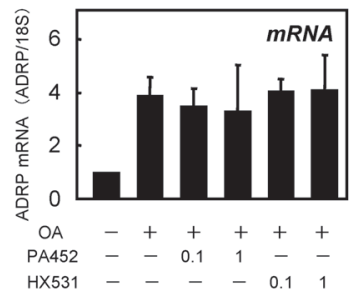

C

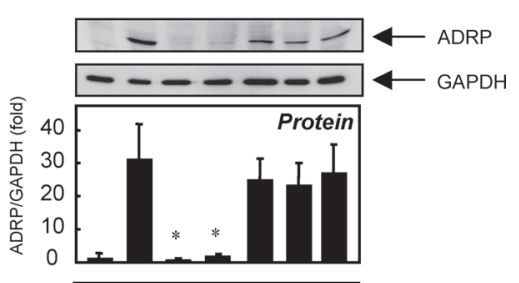

D

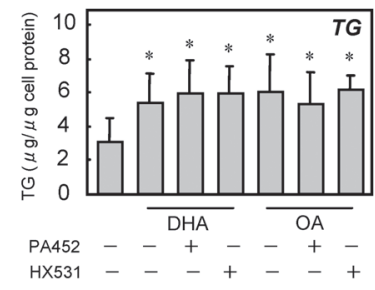

E

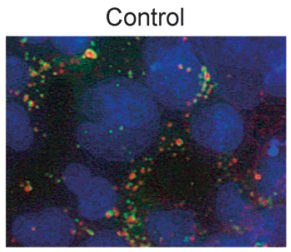

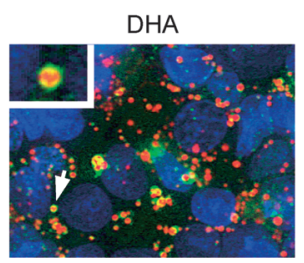

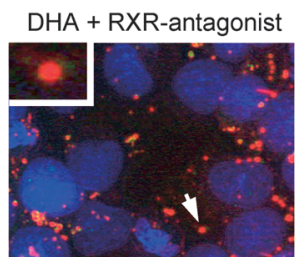

Fig. 2. Effects of RXR Antagonists on ADRP Induction in BeWo Cells

(A, B) BeWo cells were incubated for $24 \mathrm{~h}$ in medium containing $100 \mu \mathrm{M}$ DHA or OA with or without PA452 or HX531 (0.1 or $1 \mu \mathrm{M})$. Total RNA was extracted from the cells and real-time PCR experiments were carried out for ADRP and 18S. (C) After BeWo cells were incubated in medium containing $100 \mu \mathrm{M}$ DHA or OA for $24 \mathrm{~h}$ with or without $0.1 \mu \mathrm{m}$ PA452 or HX531, ADRP and GAPDH in the cell lysates were detected by immunoblotting. (D) The amounts of TG in the cell lysates were measured using the enzymatic method. (E) BeWo cells were cultured in medium containing $100 \mu \mathrm{M}$ DHA with or without $1 \mu \mathrm{M}$ PA452 for $24 \mathrm{~h}$. Cells were fixed with formaldehyde, immunostained using antiADRP antibody (yellow) and Alexa488-labeled second antibody. LD (red) and nuclei (blue) were counterstained with Oil Red O and DAPI, respectively. The inset shows a higher magnification view of the LD indicated with the arrow. * Statistically significant $(p<0.05)$ between with and without RXR-antagonist $(A-C)$ or compared to medium alone (D).

amount of ADRP was also regulated by the cellular amount of TG. ${ }^{9,10)}$

Effect of DHA Was Blocked by RXR-Antagonists DHA is one of the natural RXR-agonistic fatty acids, while OA has little reactivity to RXR. ${ }^{19,20)}$ To see whether the induction of ADRP by DHA in BeWo cells is medicated by RXR activation, we tested the effect of two RXR-antagonists, PA452 and HX531. Treatment of BeWo cells with $0.1 \mu \mathrm{M}$ PA452 or HX531 together with $100 \mu \mathrm{M}$ DHA significantly decreased the Adrp mRNA level (Fig. 2A). These RXRantagonists completely blocked DHA-induced ADRP protein accumulation (Fig. 2B). The induction of ADRP by OA, however, was not affected by the RXR-antagonists (Figs. 2B, C). These results show that DHA, but not OA, could increase Adrp transcripts through the activation of RXR. Interestingly, when BeWo cells were treated with both DHA and the RXR antagonists, TG accumulation was not affected in spite of a lack of ADRP protein (Fig. 2D), and ADRP-poor LDs were observed in the cells (Fig. 2E).

The amount of TIP47, another PAT family protein, was not changed by the addition of the fatty acids. Furthermore, the amount of TIP47 was not increased when ADRP was suppressed by the RXR antagonists (data not shown). These results suggest that TIP47 is not compensatory in BeWo cells under these conditions.

Effects of RXR Agonists on ADRP mRNA and Protein Expression To see the effects of RXR activation on ADRP protein expression, we added two specific RXR agonists, PA024 and HX630, to BeWo cells without DHA. Both agonists showed up-regulation of Adrp transcripts in BeWo cells
(Fig. 3A) but did not increase ADRP protein levels (Fig. 3B). Incubation of the cells with DHA alone induced ADRP protein concomitant with TG accumulation (Figs. 3B, C). It has been reported that ADRP is degraded by a proteasomedependent pathway when the lipid source is limited in macrophages and hepatic cells. ${ }^{9,10)}$ Thus we tested the effect of a proteasome inhibitor, MG-132, on ADRP protein expression. The addition of MG-132 alone to the cells did not induce ADRP at all (data not shown); however, induction of ADRP protein by the RXR agonists was observed in the presence of MG-132 (Fig. 3D), indicating that RXR activation is able to increase Adrp transcripts, but that ADRP protein is degraded by proteasomes without the addition of lipid sources.

Effects of PPAR $\gamma$ on ADRP mRNA and Protein Expression RXR can function as a heterodimer with the nuclear receptor superfamily. PPAR $\gamma$ is an RXR heteropartner. We next investigated the effects of a PPAR $\gamma$-antagonist, GW9662, on ADRP expression. Treatment of BeWo cells with either $100 \mu \mathrm{M}$ DHA or OA with $10 \mu \mathrm{M}$ GW9662 significantly decreased the Adrp mRNA level and completely blocked ADRP protein accumulation (Figs. 4A, B). However, GW9662 did not change the amount of TG accumulation in the presence of fatty acids in spite of decreased ADRP levels (Fig. 4C).

We also examined the effect of a PPAR $\gamma$ agonist, GW1929, on ADRP expression by DHA or OA. Treatment of BeWo cells with GW1929 alone slightly increased Adrp mRNA expression but did not increase ADRP protein levels (Figs. 4D, E). Induction of Adrp mRNA and protein by DHA 

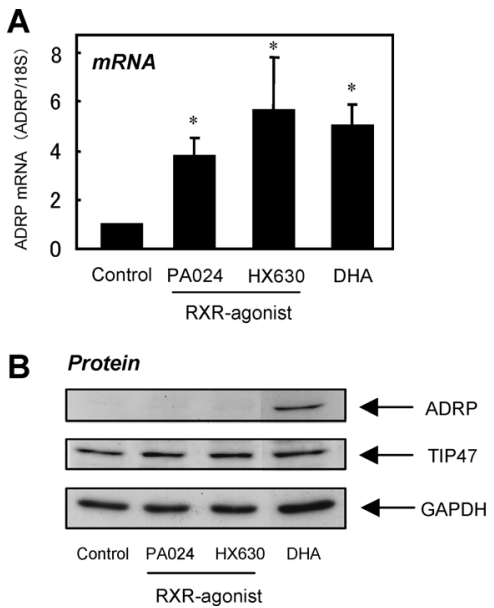
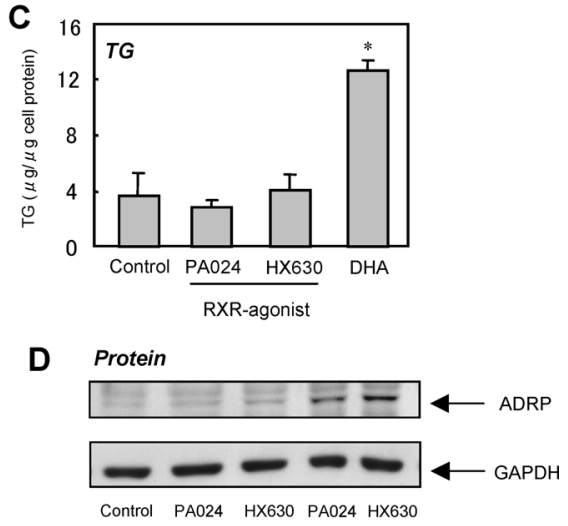

MG-132

Fig. 3. RXR Agonist Alone Did Not Induce ADRP Protein Expression

(A) BeWo cells were treated with RXR agonists, $0.1 \mu \mathrm{M}$ PA024 and $1 \mu \mathrm{M}$ HX630, in serum-containing medium without addition of fatty acids for $24 \mathrm{~h}$. Total RNA was extracted from the cells and real-time PCR experiments were carried out to evaluate the amount of mRNAs for ADRP and 18S. Values are reported as the amount of Adrp mRNA relative to 18S mRNA. (B) ADRP and GAPDH in the cell lysates were detected by immunoblotting. (C) The amounts of TG in the cell lysates were measured using the enzymatic assay. (D) BeWo cells were treated with the RXR agonists together with or without proteasome inhibitor MG-132 $(10 \mu \mathrm{M})$, for $4 \mathrm{~h}$. ADRP and GAPDH in the cell lysates were detected by immunoblotting. * Statistically significant $(p<0.05)$ compared to control (vehicle alone)

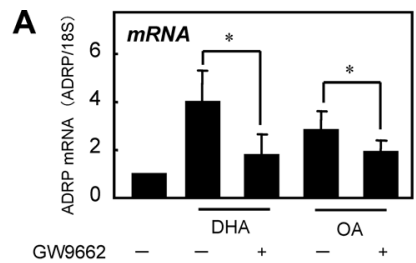

B

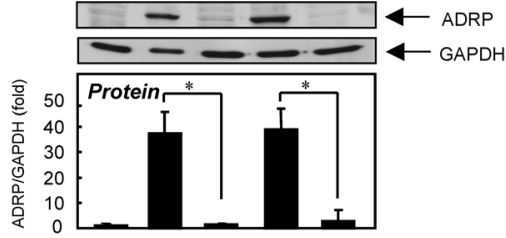

C

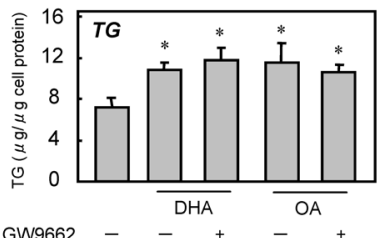

D

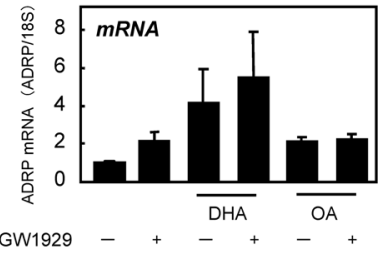

E

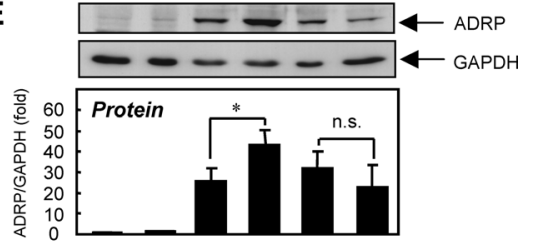

F

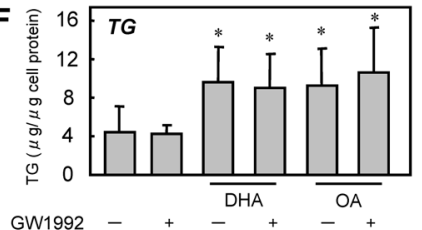

Fig. 4. Effects of an Agonist and an Antagonist of PPAR $\gamma$ on ADRP Induction in BeWo Cells

(A, D) BeWo cells were incubated in medium containing $100 \mu \mathrm{m}$ DHA or OA for $24 \mathrm{~h}$ with $10 \mu \mathrm{M}$ GW9662 (PPAR $\gamma$-antagonist) or $2 \mu \mathrm{m}$ GW1929 (PPAR $\gamma$-agonist). Total RNA was extracted from the cells and real-time PCR experiments were carried out for ADRP and 18S. Values are reported as the amount of Adrp mRNA relative to 18S mRNA. (B, E) After BeWo cells were incubated in medium containing $100 \mu \mathrm{M}$ DHA or OA for $24 \mathrm{~h}$ with $10 \mu \mathrm{M}$ GW9662 (PPAR $\gamma$-antagonist) or $2 \mu \mathrm{M}$ GW1929 (PPAR $\gamma$-agonist), ADRP and GAPDH in the cell lysates were detected by immunoblotting. * Statistically significant $(p<0.05)$ between with and without PPAR $\gamma$-antagonist (A, B) or agonist (D, E). n.s.: not significant. (C, F) Under the same experimental conditions, the amounts of TG in the cell lysates were measured using the enzymatic assay. * Statistically significant $(p<0.05)$ compared to medium alone.

was enhanced by GW1929, but OA-induced Adrp mRNA and protein expression was not. However, no effect was observed on the amounts of TG by the agonist (Fig. 4F). These results together with others indicate that the DHA increases ADRP levels through activation of RXR/PPAR $\gamma$ heterodimer, and OA increased ADRP levels through PPAR $\gamma$ activation independent of RXR.

\section{DISCUSSION}

Recent studies have shown that LD is a ubiquitously expressed orgalelle related to lipid homeostasis, and that ADRP and TIP47, two major PAT family proteins, are present in placenta. ${ }^{1,25)}$ DHA is not just an important nutrient for fetal development but is proposed to be a natural RXR agonist. ${ }^{19,20)}$ Here we demonstrated that DHA, but not OA, functions as an RXR activator to induce ADRP expression in human choriocarcinoma cell line BeWo cells.

DHA-induced expression of ADRP protein in BeWo cells was significantly suppressed both by the RXR antagonists, PA452 and HX531, and by the PPAR $\gamma$ antagonist, GW9662. Therefore it is likely that ADRP increases through transcriptional activation of the RXR/PPAR $\gamma$ heterodimer. Some recent reports have suggested that ADRP gene transcription is up-regulated through PPAR-response elements by PPARs and RXR. ${ }^{6,25,26)}$ Bildirici et al. reported that RXR agonists 
induce ADRP protein expression in human placental trophoblasts. $\left.{ }^{6}\right)$ Our current data correspond well with these observations, and further confirmed the RXR-dependent responses with experiments using RXR antagonists. In addition, we showed that DHA, but not OA, acts as a natural agonist of RXR. We note that the OA-induced response was not RXR-dependent but PPAR $\gamma$-dependent, since the antagonist for PPAR $\gamma$, but not RXR, blocked OA-induced ADRP expression. de Urquiza et al. showed that DHA and arachidonic acid but not OA activate RXR in in vitro reporter gene assay. ${ }^{19)}$ On the other hand, Auwerx reported that DHA bind and activate PPAR $\gamma .{ }^{27)}$ It is likely that the DHA increases ADRP expression through both activation of RXR-homodimer and RXR/PPAR $\gamma$ heterodimer in BeWo cells.

DHA is required for fetus development during pregnancy. The proportion of DHA in fetal plasma was shown to be higher than maternal plasma, and DHA concentration in the plasma from maternal vein blood was $41.9 \pm 20.9 \mu \mathrm{g} / \mathrm{ml} .{ }^{15}$ ) Since the molecular weight of the DHA is $328.5,100 \mu \mathrm{M}$ of DHA used in this study is equal to $32.9 \mu \mathrm{g} / \mathrm{ml}$. Thus, our experimental condition could reflect the maternal circulation. It has been speculated that DHA-dependent induction of ADRP is of physiological importance. DHA is most effectively taken up by fetal cells, whereas human hepatoma cell lines HepG2 and HuH-7 show no preference for fatty acid species. ${ }^{2829)}$ DHA-dependent induction of ADRP was far more sensitive to the RXR antagonists in BeWo cells than in $\mathrm{HuH}-7$ cells, which required about $10 \mu \mathrm{M}$ (data not shown). Certainly more studies are needed to elucidate the physiological relevance of DHA-dependent RXR activation during pregnancy, but our study opens up the possibility that regulation of lipid metabolism in placental development could be a useful target.

In this study, DHA increases cellular TG level in BeWo cells and it is previously reported that DHA raises TG accumulation in other cells, such as hepatocyte. ${ }^{30)}$ However, in 3T3-L1 preadipocytes, DHA suppressed cellular lipid accumulation during adipocyte differentiation, ${ }^{31)}$ suggesting that the effect of the DHA is different by cell types.

In our experiments, we observed ADRP-poor LD formation by suppression of ADRP by RXR antagonists. It is thought that LD should be covered and stabilized by some proteins such as PAT family proteins. Chan et al. reported that LD formed in the liver of ADRP-null mice is enriched with another LD-coating protein, TIP47. ${ }^{32}$ It is interesting that TIP47 was not up-regulated when the ADRP-poor LD was formed in BeWo cells by the RXR antagonists. There might be some other LD-coating proteins on the ADRP-poor LD particles.

We note that induction of Adrp mRNA does not directly correlate to ADRP protein levels. The fatty acids itself are not only substrates for TG biosynthesis but also the regulation as the transcription factor activity. Our results suggest that the bifunctional of the fatty acids are necessary for ADRP stabilization. We and others have shown that ADRP protein can be degraded by a proteasome-dependent pathway in macrophages and hepatic cells when cellular amount of TG is poor. ${ }^{9,10)}$ Our data suggest that similar post-translational regulation could occur in BeWo cells, since results with the proteasome inhibitor MG-132 suggest that ADRP protein synthesis should have been enhanced by addition of the RXR agonists.

In conclusion, DHA acts as a natural ligand of RXR and increases ADRP expression in BeWo cells. The amount of ADRP in the cells depends not only on the transcriptional level, but also on the availability of lipids.

Acknowledgements We would like to thank M. Konishi, S. Fukagawa, and H. Hasegawa for their technical assistance. This work was supported in part by a Showa University Grant-in Aid for Innovative Collaborative Research Projects, Research on Publicly Essential Drugs and Medical Devices from the Japan Health Sciences Foundation, and Grants-inAid for Scientific Research (19590076) from MEXT of Japan.

\section{REFRENCES}

1) Murphy D. J., Vance J., Trends Biochem. Sci., 24, 109-115 (1999).

2) Murphy D. J., Prog. Lipid Res., 40, 325-438 (2001).

3) Fujimoto Y., Itabe H., Sakai J., Makita M., Noda J., Mori M., Higashi Y., Kojima S., Takano T., Biochim. Biophys. Acta, 1644, 47-59 (2004).

4) Liu P., Ying Y., Zhao Y., Mundy D. I., Zhu M, Anderson R. G. W., J. Biol. Chem., 279, 3787-3792 (2004).

5) Ackerman W. E. 4th., Robinson J. M., Kniss D. A., Placenta, 28, 465-476 (2007).

6) Bildirici I., Roh C. R., Schaiff W. T., Lewkowski B. M., Nelson D. M., Sadovsky Y., J. Clin. Endocrinol. Metab., 88, 6056-6062 (2003).

7) Tobin K. A., Harsem N. K., Dalen K. T., Staff A. C., Nebb H. I., Duttaroy A. K., J. Lipid Res., 47, 815-823 (2006).

8) Gao J., Serrero G., J. Biol. Chem., 274, 16825-16830 (1999).

9) Masuda Y., Itabe H., Odaki M., Hama K., Fujimoto Y., Mori M., Sasabe N., Aoki J., Arai H., Takano T., J. Lipid Res., 47, 87-98 (2006).

10) Xu G., Sztalryd C., Lu X., Tansey J. T., Gan J., Dorward H., Kimmel A. R., Londos C., J. Biol. Chem., 280, 42842-42847 (2005).

11) Lairitzen L., Hansen H. S., Jorgensen M. H., Michaelsen K. F., Prog. Lipid Res., 40, 1-94 (2001).

12) Uauy R., Hoffman D. R., Peirano P., Birch D. G., Birch E. E., Lipids, 36, 885-895 (2001).

13) Larque E., Demmelmair H., Koletzko B., Ann. N. Y. Acad. Sci., 967, 299-310 (2002).

14) Campbell F. M., Gordon M. J., Dutta-Roy A. K., Mol. Cell. Biochem., 155, 77-83 (1996).

15) Ruyle M., Connor W. E., Anderson G. J., Lowensohn R. I., Proc. Natl. Acad. Sci. U.S.A., 87, 7902-7906 (1990).

16) Barak Y., Nelson M. C., Ong E. S., Jones Y. Z., Ruiz-Lozano P., Chien K. R., Koder A., Evans R. M., Mol. Cell, 4, 585-595 (1999).

17) Sapin V., Dolle P., Hindelang C., Kastner P., Chambon P., Dev. Biol., 191, 29-41 (1997).

18) Schaiff W. T., Carlson M. G., Smith S. D., Levy R., Nelson D. M., Sadovsky Y., J. Clin. Endocrinol. Metab., 85, 3874-3881 (2000).

19) de Urquiza A. M., Liu S., Sjoberg M., Zetterstrom R. H., Griffiths W., Sjovall J., Perlmann T., Science, 290, 2140-2144 (2000).

20) Lengqvist J., de Urquiza A. M., Bergman A. C., Willson T. M., Sjovall J., Perlmann T., Griffiths W., Mol. Cell. Proteomics, 3, 692-703 (2004).

21) Umemiya H., Fukasawa H., Ebisawa M., Eyrolles L., Kawachi E., Eisenmann G., Gronemeyer H., Hashimoto Y., Shudo K., Kagechika H., J. Med. Chem., 40, 4222-4234 (1997).

22) Ohta K., Kawachi E., Inoue N., Fukasawa H., Hashimoto Y., Itai A., Kagechika H., Chem. Pharm. Bull., 48, 1504-1513 (2000).

23) Ebisawa M., Umemiya H., Ohta K., Fukasawa H., Kawachi E., Christoffel G., Gronemeyer H., Tsuji M., Hahimoto Y., Shudo K., Kagechika H., Chem. Pharm. Bull., 47, 1778-1786 (1999).

24) Takahashi B., Ohta K., Kawachi E., Fukasawa H., Hashimoto Y., Kagechika H., J. Med. Chem., 45, 3327-3330 (2002).

25) Gerhold D. L., Liu F., Jiang G., Li Z., Xu J., Lu M., Sachs J. R., Bagch A., Fridman A., Holder D. J., Doebber T. W., Berger J., Elbrecht A., Moller D. E., Zhang B. B., Endocrinology, 143, 2106-2118 (2002).

26) Nadra K., Anghel S. I., Joye E., Tan N. S., Basu-Modak S., Trono D. 
Wahli W., Desvergne B., Mol. Cell. Biol., 26, 3266-3281 (2006).

27) Auwerx J., Diabetologia, 42, 1033-1049 (1999).

28) Campbell F. M., Clohessey A. M., Gordon M. J., Dutta-Roy A. K., J. Lipid Res., 38, 2558-2568 (1997).

29) Fujimoto Y., Onoduka J., Homma K. J., Yamaguchi S., Mori M., Higashi Y., Makita M., Kinoshita M., Noda J., Itabe H., Takano T., Biol. Pharm. Bull., 29, 2174-2180 (2006).
30) Granlund L., Larsen L. N., Nebb H. I., Pedersen J. I., Biochim. Biophys. Acta, 1687, 23-30 (2005).

31) Kim H.-K., Della-Fera M., Lin J., Baile C. A., J. Nutr., 136, 29652969 (2006)

32) Chang B. H., Li L., Paul A., Taniguchi S., Nannegari V., Heird W. C., Chan L., Mol. Cell. Biol., 26, 1063 -1076 (2006). 